\section{Self-reports of executive functioning in multiple sclerosis: to trust or not to trust}
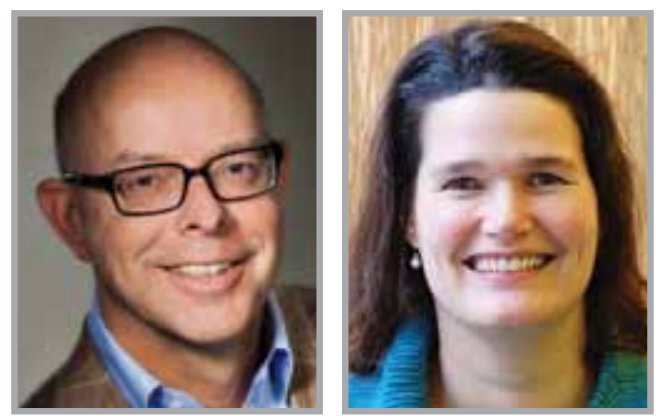

\author{
“In early relapsing-remitting \\ multiple sclerosis, the incidence \\ of cognitive symptoms is 30\%, \\ and 4 years after diagnosis one \\ out of three relapsing-remitting \\ multiple sclerosis patients has \\ cognitively declined."
}

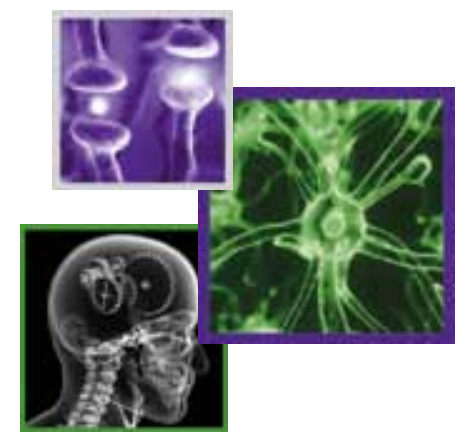

Leo HVisser ${ }^{* 1,2} \&$ Karin van der Hiele Hi,4 $^{3,4}$

Cognitive impairment is a disabling symptom in multiple sclerosis (MS), occurring in $45-65 \%$ of the patients [1]. It involves information processing speed, complex attention, (episodic) memory and executive functions. In early relapsing-remitting MS, the incidence of cognitive symptoms is $30 \%$, and 4 years after diagnosis one out of three relapsing-remitting MS patients has cognitively declined. In this relatively young patient group, it has been shown that worsening of cognitive symptoms and a decline on neuropsychological testing over time are predictive of a deterioration in employment status.

The morbidity associated with MS will gradually increase as a result of these cognitive deficits, with adverse effects relating to the patient's ability to perform basic daily tasks, to work, socialize, form relationships and pursue recreational activities [2].

Therefore, early, accurate detection of cognitive dysfunction is imperative, enabling strategies to be put in place to compensate these associated limitations. The best way to elicit cognitive difficulties is with neuropsychological testing. However, neuropsychological assessment is time consuming and may not always be easily accessible. A screening tool would be ideal to assess which patients need more extensive neuropsychological assessment $[2,3]$. The question is whether self-reports of cognitive performance can be useful to address this issue. How accurate are patients with MS when it comes to assessing their own cognition?

In the literature, there is a debate on whether self-reports are adequate for assessing cognitive impairment in MS patients. The relationship between subjective (self-reported) cognitive performance and objective or informant-rated cognitive performance may be mediated by psychological factors such as depression, other neuropsychiatric symptoms and coping mechanisms [3]. Whereas underestimated cognitive performance has been associated with depressive symptoms, overestimation, however, has been linked with less depression, conscientiousness, an increase of $\operatorname{cog}$ nitive impairment, disinhibited euphoric behavior and underemployment. From a group of 718 patients we selected 128 
“...patients and caregivers will benefit by receiving accurate information concerning their perceived and actual cognitive and psychological functioning, leading to appropriate suggestions for further therapeutic interventions.” patients, half of them reported no executive function disturbances and half of them did [3]. Both patient groups underwent extensive neuropsychological testing and the psychological characteristics of MS patients that under-, overor accurately estimated their executive performance were examined. Approximately $70 \%$ of the patients assessed their executive functioning correctly, they did not have a discrepancy between self-reported and actual executive functioning. Almost 15\% overestimated their executive performance. They did not report impairments, but showed executive dysfunction on testing. A further $15 \%$ underestimated their executive performance, reported executive function disturbances but showed normal executive performance. Characteristics of patients underestimating their cognitive performance were increased depression, anxiety and psychological stress. They frequently exhibited the 'disclosure of emotions' style of coping and displayed a more passive reaction pattern [3].

A slight slowing in their rate of information processing and decreased cognitive flexibility, compared with accurate estimators, was shown by MS patients who underestimated their executive abilities. It can therefore occur that in more strenuous situations, for example, demanding vocational settings, cognitive dysfunctions that went unnoticed on neuropsychological testing do become evident for the patient and therefore they report these dysfunctions. In this respect, executive complaints should always be taken seriously. Previous studies on aging even revealed that memory complaints in elderly individuals were predictive of future cognitive decline and dementia [4]. Considering that exact relationships remain unclear, more psychological distress and a negative report basis could be the result of slight cognitive disability awareness. Discrepancies between subjective and objective cognitive performance could be influenced by 'coping mechanisms'. Where discrepancies between objective and subjective amounts of impairments are concerned, it has been suggested that adaptive or maladapted coping mechanisms (including denial, lack of insight or overestimation) and not depressive symptoms may be responsible [5].

For clinicians, information from partners or caregivers is vital in those who underestimated their cognitive performance. A considerable decrease of underestimators occurred when informant-based reports were used [3].
This study showed no difference between overestimators, and accurate estimators in depression, anxiety, psychological stress or coping styles. Furthermore, no increases in accuracies was apparent by using informant-based reports. Expanded Disability Status Scale scores were higher (although not significant) in the overestimator groups [3].

The characterizations of overestimators in previous studies were psychological features bearing resemblance to euphoria sclerotica, and a reason for not noticing cognitive disturbances could be the fact that less cognitively challenging activities are demanded by the social environment, leading both themselves and informants to the false belief of being cognitively intact. It has been suggested that lack of insight in MS displayed by overestimators was the result of diffused brain pathology with disproportionate atrophy of the prefrontal cortex [6]. It has been acknowledged that there is a link between greater physical disability and more behavioral problems, such as euphoria and an increase in frontal brain pathology, which could be contributing factors to overestimating cognitive functioning.

Although their identification is important for treatment purposes, previous studies did not discriminate between under- and over-estimators. Moreover, rarely investigated is the influence of psychological stress and coping methods on the accuracy of self-reports. This knowledge may have important consequences for the psychological treatment and rehabilitation of MS patients.

So, when patients are complaining of executive problems, check for depression and anxiety. If absent, the probability of the patient's complaints proving to be accurate, increases. On the other hand, self-assessment of cognition by a patient presenting with more advanced progressive disease linked to greater physical disability, with disinhibiting or euphoric behavior, reporting absence of cognitive disabilities should be considered unreliable $[2,3]$. In either situation, a neuropsychological examination will provide the final answer.

The above reflects a clinician's point of view. But what about the patient perspective? The patient reports a problem in cognitive function. Perceived processing speed difficulties have been associated with impulsivity, slowed motor speed, greater anxiety and increased introversion [7]. Evaluation of anxiety and patient education about objective measures versus subjective perceptions of slowed processing speed may be 
helpful to MS patients who report significant cognitive slowing [7]. It is important to address this issue, as these perceived problems may lead to feelings of uncertainty in both patients and caregivers. It is of importance to ask the patient for any limitations in daily life, in their work situation and in their relationships with others. For the patient there is no discussion to trust or not to trust his or her voice and perception. It can be reassuring for the patient that cognitive complaints are not always related to cognitive dysfunction and to realize that anxiety or depression may play a role in both perceived and actual cognitive impairment. Patients and caregivers unaware of the patient's cognitive dysfunction may benefit from understanding the nature and impact of these impairments, creating clarity about everyday problems they may have encountered.

Addressing these issues may help the patient to cope with these complaints and improve selfefficacy. The patient can also adapt to the presence of cognitive impairment and limit her- or him-self in difficult cognitive tasks.

\section{References}

1 Chiaravalloti ND, DeLuca J. Cognitive impairment in multiple sclerosis. Lancet Neurol. 7(12), 1139-1151 (2008).

2 Feinstein A. Multiple sclerosis and cognitive dysfunction: how accurate are patients' self-assessments? Eur. J. Neurol. 19(4), 535-536 (2012).

3 van der Hiele K, Spliethoff-Kamminga NG, Ruimschotel RP, Middelkoop HA, Visser LH. The relationship between self-reported
From a clinician's perspective, we may conclude that self-reports of executive functioning are valuable, but should be viewed with caution as they may not always be accurate. When used in combination with an initial neuropsychological screening we may identify patients in need for further assessment based on their cognitive or psychological profile. Ultimately, patients and caregivers will benefit by receiving accurate information concerning their perceived and actual cognitive and psychological functioning, leading to appropriate suggestions for further therapeutic interventions.

Financial \& competing interests disclosure

The authors have no relevant affliations or financial involvement with any organization or entity with a financial interest in or financial conflict with the subject matter or materials discussed in the manuscript. This includes employment, consultancies, honoraria, stock ownership or options, expert testimony, grants or patents received or pending, or royalties.

No writing assistance was utilized in the production of this manuscript. executive performance and psychological characteristics in multiple sclerosis. Eur. J. Neurol. 19(4), 562-569 (2012).

4 Wang L, van BG, Crane PK et al. Subjective memory deterioration and future dementia in people aged 65 and older. J. Am. Geriatr. Soc. 52(12), 2045-2051 (2004).

5 Gold SM, Schulz H, Monch A, Schulz KH, Heesen C. Cognitive impairment in multiple sclerosis does not affect reliability and validity of self-report health measures. Mult. Scler. 9(4), 404-410 (2003).
6 Carone DA, Benedict RH, Munschauer FE III, Fishman I, Weinstock-Guttman B. Interpreting patient/informant discrepancies of reported cognitive symptoms in MS. J. Int. Neuropsychol. Soc. 11(5), 574-583 (2005).

7 Roberg BL, Bruce JM, Lovelace CT, Lynch S. How patients with multiple sclerosis perceive cognitive slowing. Clin. Neuropsychol. 26(8), 1278-1295 (2012). 